\title{
Infection of the central nervous system with dengue virus 3 genotype I causing neurological manifestations in Brazil
}

\author{
Danilo Bretas de Oliveira ${ }^{[1]}$, Guilherme Machado ${ }^{[2]}$, Gabriel Magno de Freitas Almeida ${ }^{[1]}$, \\ Paulo César Peregrino Ferreira' ${ }^{[1]}$, Cláudio Antônio Bonjardim ${ }^{[1]}$, \\ Giliane de Souza Trindade ${ }^{[1]}$, Jônatas Santos Abrahão ${ }^{[1]}$ and Erna Geessien Kroon ${ }^{[1]}$
}

[1]. Universidade Federal de Minas Gerais, Belo Horizonte, Minas Gerais, Brasil. [2]. Hospital Risoleta Tolentino Neves, Belo Horizonte, Minas Gerais, Brasil.

\begin{abstract}
A case of dengue virus 3 (DENV-3) genotype I infection with neurological manifestations occurred in Belo Horizonte, Minas Gerais in October 2012. The serotype was detected by PCR, and the genotype was assessed by sequencing and phylogenetic analysis of the C-prM region. The virus causing neurological manifestations clustered with other sequences of DENV-3 genotype I. Because neurological manifestations of DENV are possibly misdiagnosed in Brazil, this study serves as an alert of the importance of DENV diagnoses in CNS infections.
\end{abstract}

Keywords: Central nervous system. Neurologic manifestations of dengue virus. Dengue virus 3 genotype I.

\section{INTRODUCTION}

Dengue virus (DENV) is the most important mosquito-borne disease in humans. Approximately 2.5 billion people worldwide live in areas with a risk of dengue transmission, and it is estimated that 50 million dengue infections occur annually ${ }^{(1)(2)}$. In Minas Gerais, Brazil (Figure 1), 21,105cases were reported in 2012, and more than 350,000 cases were reported in 2013 $(1,750 \text { cases } / 100,000 \text { habitants })^{(3)}$.

Different DENV serotypes $(1,2,3,4)$ are usually associated with systemic and dynamic diseases, with clinical illness ranging from a nonspecific viral syndrome (dengue fever with or without warning signs) to severe disease (severe dengue). Dengue is classified as severe when the disease progresses to severe plasma leakage, hemorrhage, and organ (e.g., CNS) impairment ${ }^{(2)}$. Among the plethora of clinical symptoms caused by DENV, neurological manifestations are often misdiagnosed or neglected. During dengue epidemics in Brazil, public health organizations and clinics are typically overloaded, and within this context, some atypical or rare dengue clinical manifestations are not properly diagnosed ${ }^{(2)(4)}$.

Phylogenetic studies have classified DENV-3 into five genotypes (I, II, III, IV, and V) based on their genetic diversity ${ }^{(5)}$. Our laboratory has isolated and characterized DENV-3 genotype I from patients and mosquitoes in different cities of Minas Gerais (Figure 1), most recently (2013) in Contagem, MG

Corresponding author: Dra. Erna Geessien Kroon.

e-mail: kroone@icb.ufmg.br I ernagkroon@gmail.com

Received 27 June 2015

Accepted 19 August 2015 (unpublished data $)^{(6)(7)}$. The patients presented with a wide range of clinical manifestations including headache, light sensitivity, retro-orbital pain, general body pain (arthralgia, myalgia), vomiting, and hemorrhage ${ }^{(6)}$. In addition, previous studies have demonstrated an important feature of DENV-3 genotype I, which is its ability to cause CNS infection and modulate the immunological system in a mouse model that was intracranially inoculated ${ }^{(8)(9)}$.Neurological manifestations of dengue infection in humans range from a common headache to more severe symptoms, such as nuchal rigidity, reduced consciousness, and convulsions. Moreover, some cases can experience severe encephalopathy, which can lead to sequelae ${ }^{(10)}$.

\section{CASE REPORT}

Our laboratory was contacted by a local hospital to diagnose a 21-year-old patient in October 2012. On October 1, she presented with the onset of dengue symptoms, including fever, myalgia, and general malaise. On October 7, the hospital emergency department attended the patient, who presented with generalized seizures, and released her on the same day. On October 9, the patient presented with another episode of seizures and was admitted to the hospital to be treated with an anticonvulsant. An episode of seizures occurred on the following day, and an electroencephalogram revealed irritation in the frontal region of the brain. The last episode of seizures was described on October 13, when the patient received intensive care at the hospital and cerebrospinal fluid (CSF) was collected. The results revealed the presence of leukocytes $(7$ cells $/ \mathrm{mL})$, glucose levels in the CSF of $78 \mathrm{~g} / \mathrm{L}$, and protein levels of $36 \mathrm{~g} / \mathrm{L}$. The polymerase chain reaction (PCR) to detect Human herpes virus 1 and 2 (HSV-1 and HSV-2), Human herpesvirus 3 [varicella 


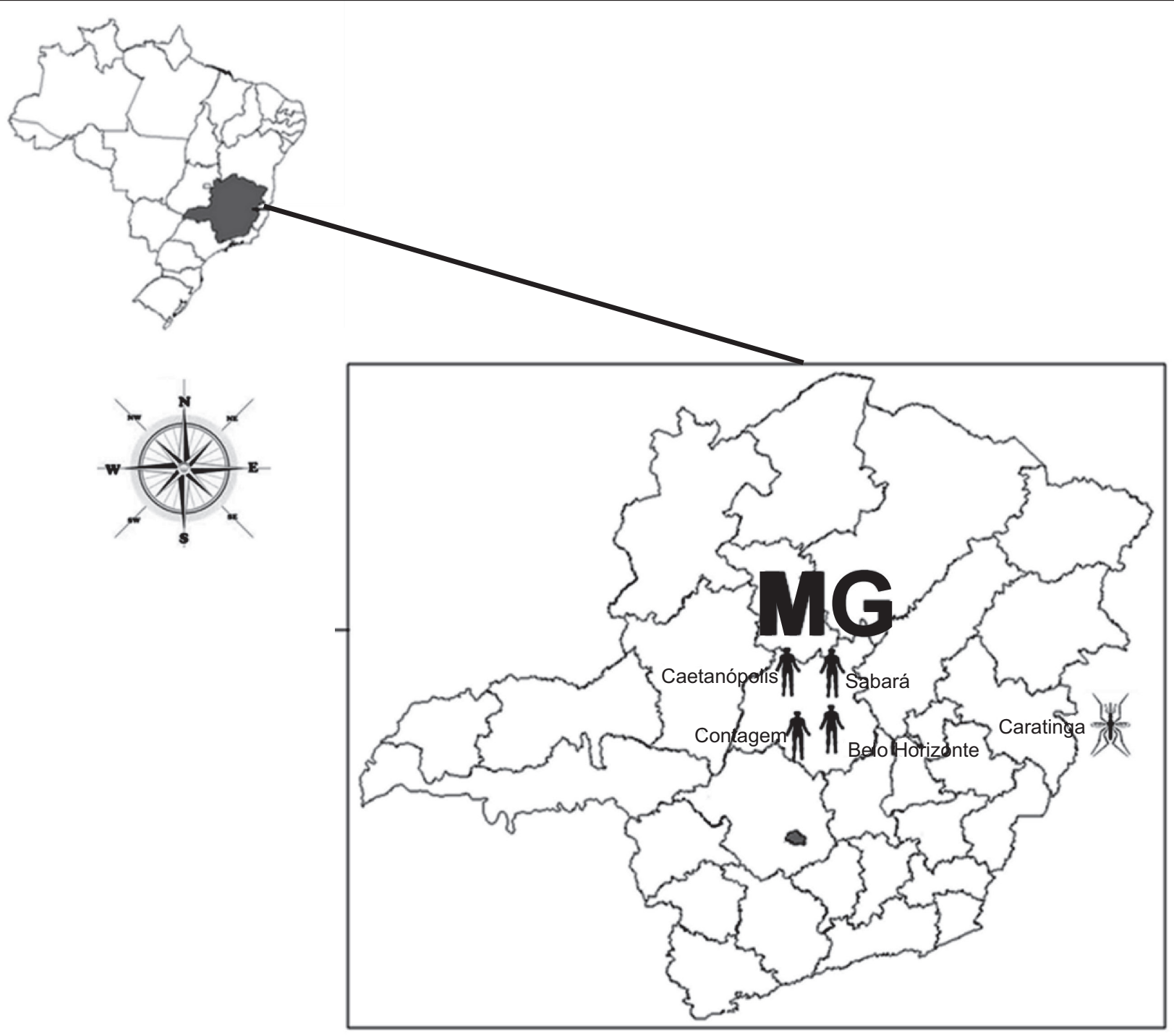

FIGURE 1 - Map of dengue virus 3 (DENV-3) genotype I distribution. Map of Minas Gerais indicating the cities where DENV-3 genotype I was detected. Human symbol= human cases: Belo Horizonte [2003-dengue fever (DF)]; 2004, dengue hemorrhagic fever-DHF; 2012 dengue infection with neurologic manifestations) $)^{(6)}$; Caetanópolis (2002-DF) ${ }^{(6)}$; Contagem (2013-DF) (not published); Sabará (2003-DF) ${ }^{(6)}$. Mosquito symbol=detection in Aedes aegypti mosquitoes: Caratinga (2005 and 2006) ${ }^{(7)}$.

zoster virus) (VZV)], Human herpesvirus 5 [cytomegalovirus (CMV)] and viruses of the genus Enterovirus was negative. Bacterial culture was also negative. For the next two weeks, the patient exhibited confusion and behavioral alterations. She left the hospital on October 27 (Figure 2).

Aiming to characterize the etiological agent, the patient's CSF was sent to our laboratory. RNA was extracted (Viral RNA QIAamp Extraction Kit; Qiagen, Valencia, USA), followed by reverse transcription using random primers (MMLV; Promega, Madison, USA). The cDNA was used as the template in the quantitative PCR (qPCR) with primers designed to amplify the NS5 region of the viruses from the genus Flavivirus( ${ }^{(1)}$. qPCR was performed with a commercial mix (SYBR® Green
PCR Master Mix, Life Technologies, Carlsbad, USA), and specific amplification with a melting temperature of $79.5^{\circ} \mathrm{C}$ was observed. The primers used for amplification were forward, $5^{\prime}-$ GTGTCCCAGCCGGCGGTGTCATCAGC-3', and reverse, 5'-TACAACATGATGGGAAAGCGAGAGAAAAA-3'(11). PCR failed to detect HSV-1, HSV-2, VZV, CMV, and viruses of the genus Enterovirus.

To identify the Flavivirus that was the etiological agent of this infection, a specific PCR for DENV was performed, targeting the C-prM region (forward, 5 '-TCAATATGCTGAAACGCGCGAGAAACCG-3', and reverse, $5^{\prime}$-TTGCACCAACAGTCAATGTCTTCAGGTTC-3' ${ }^{(12)}$. Amplification of a 511-bpDNA fragment was observed, confirming 


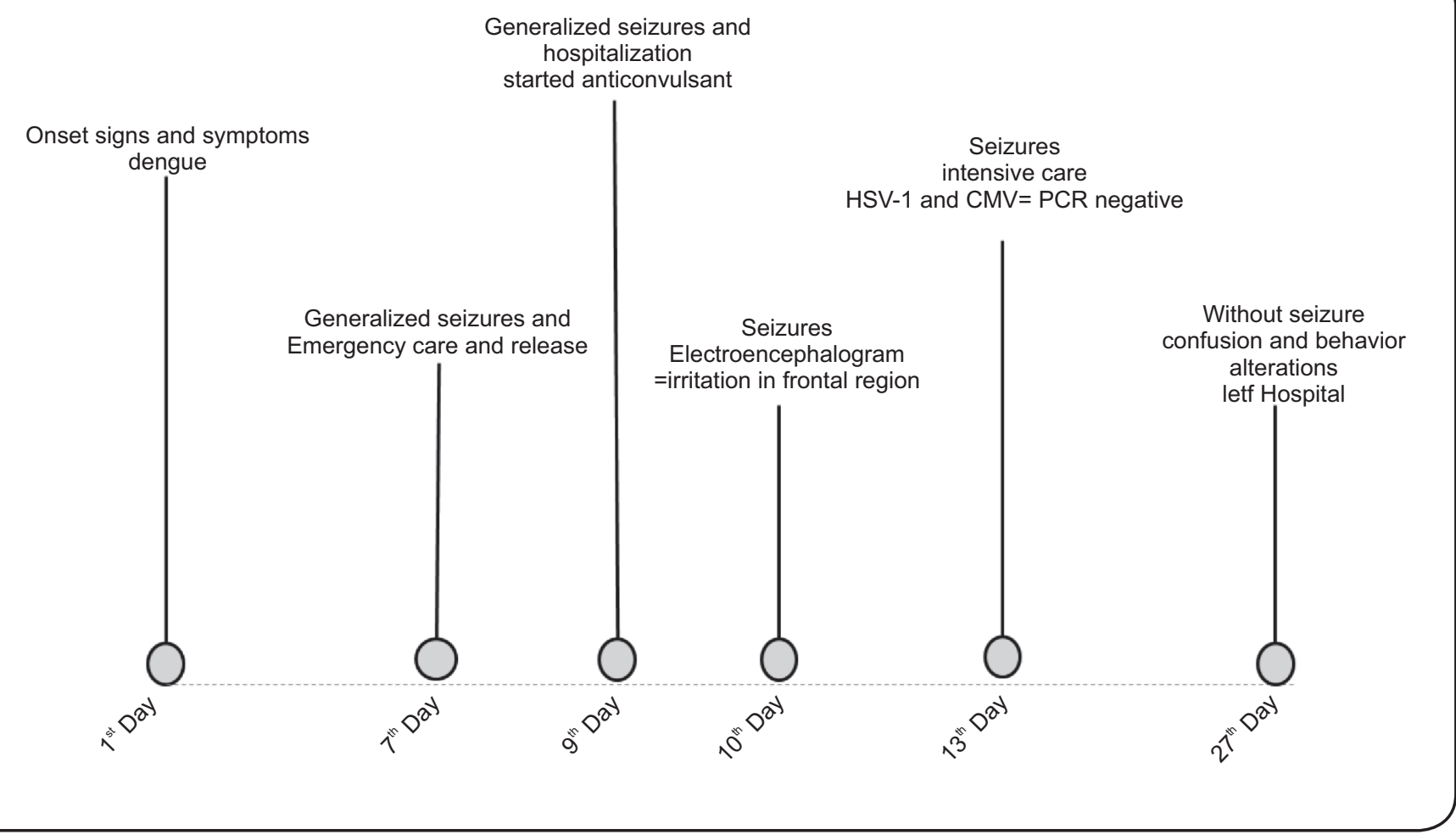

FIGURE 2 - Timeline of the dengue virus 3 genotype I infection disease course, including signs, symptoms, and clinical management. CMV: human herpes virus 5 (cytomegalovirus); HSV- 1: human herpes virus 1; PCR: polymerase chain reaction.

DENV as the etiological agent. Both PCR tests were performed in duplicate, with two different samples to confirm the diagnosis. The amplified DNA fragment was directly sequenced in both directions using a Mega-BACE-sequencer (GE Healthcare; Little Chalfont, UK) ${ }^{(13)}$.The optimal alignment of the C-prM region using ClustalW (www.ncbi.nlm.nih.gov/pmc/articles/PMC308517) and MEGA version 5 (www.megasoftware.net) showed high identity among the nucleotide sequence of the sample (GenBank accession number 1735167) and other DENV-3 sequences deposited in GenBank. The paired nucleotide identity between our sample and other DENV-3 sequences ranged from $93.8 \%$ to $98.2 \%{ }^{(14)}$. Phylogenetic trees of the C-prM region were constructed using the neighbor-joining and maximum parsimony methods with 1,000 bootstrap replicates and the Kimura 2-parameter model ${ }^{(14)}$. Both models showed that the DNA fragment sequence clustered with other sequences of DENV-3genotype I from Brazil, Asia, and Central America (Figure 3). The nucleotide identity ranged from $97.1 \%$ to $98.2 \%$ when comparing pairwise our sample with other genotype I sequences. The study followed the rules of the Ethics Committee of Universidade Federal de Minas Gerais (Number 2129.0.000.410-10).

\section{DISCUSSION}

Although data concerning the role of viral agents in CNS infections have increased in recent years, DENV has not been included in diagnostic assays because it is not considered a classical neuropathogenic virus ${ }^{(2)}{ }^{(10)}(15)$. The most frequently described neurological symptom associated with dengue is mental confusion, which is often interpreted as a consequence of high fever. CNS DENV-3 infections have been described in humans and are also reproducible in animal models ${ }^{(9)(15)}$.

Our report describes a DENV infection in the CNS caused by DENV-3 genotype I, which has also been detected in human cases and mosquitos (Figure 1) ${ }^{(6)(7)(8)}$. As DENV-3 genotype I was detected in different years in the same city (Belo Horizonte: 2002, 2003, and 2004), this genotype has been circulating in this region since that time ${ }^{(6)}$. Our previous studies demonstrated that DENV-3 genotype I had neurovirulent potential in a murine model ${ }^{(8)}$. We have now confirmed the presence of DENV-3 genotype I in the CSF of a patient with generalized seizures and behavioral changes, emphasizing the importance of this genotype to public health in Minas Gerais ${ }^{(3)}$. Because DENV infections in the CNS and the associated neurological manifestations are possibly misdiagnosed in Brazil (e.g., meningoencephalitis and encephalitis), this report serves as an alert regarding the importance of DENV diagnosis in CNS infections. Furthermore, because this viral pathogen is not identified in most cases of CNS viral infection in Brazil, the number of cases and impact on public health might be more important than suspected, and the number of dengue infections in the CNS might be underestimated ${ }^{(2)}(10)$. Finally, this study 


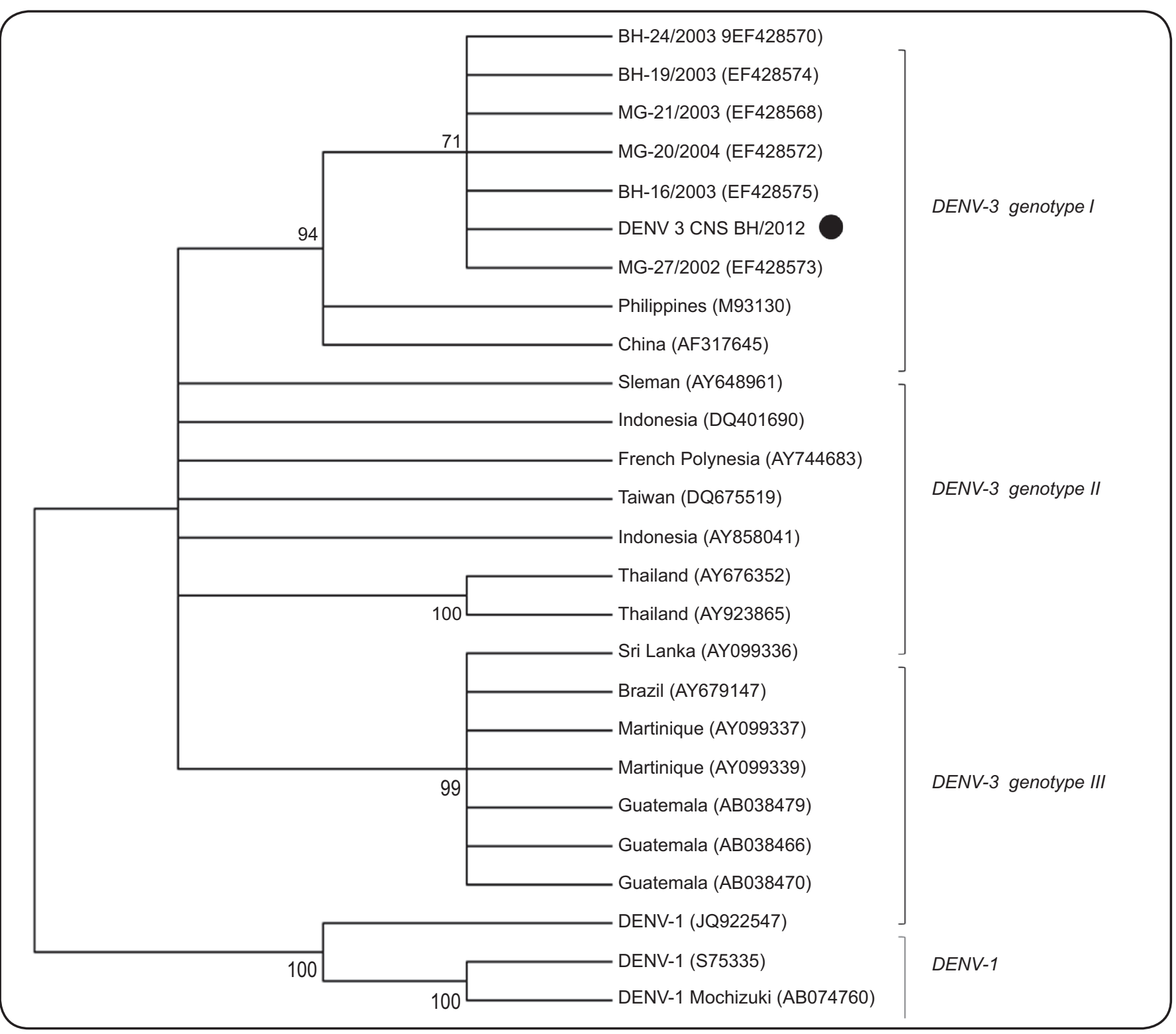

FIGURE 3 - Phylogenetic analysis of the C-prM region of dengue virus (DENV). Phylogenetic analysis of the C-prM of DENV (451bp). DENV-3 sequences deposited in GenBank were analyzed using the neighbor-joining method with the Kimura 2-parameter algorithm in MEGA5 (www.megasoftware.net). DENV-1 was used as an external group. The rate of variation among sites was modeled with a gamma distribution (shape parameter $=1$ ). Bootstrap confidence limits (from 1,000 replicates) are indicated at each node.

provides new information regarding the symptoms and signs of dengue infection in the CNS with neurological manifestations and an update to the DENV-3 genotype I epidemiology in Brazil.

\section{ACKNOWLEDGMENTS}

We thank João Rodrigues dos Santos and colleagues from the Laboratório de Vírus for their excellent technical support.

\section{CONFLICT OF INTEREST}

The authors declare that there is no conflict of interest.

\section{FINANCIAL SUPPORT}

This study was supported by grants from CNPq, CAPES, FAPEMIG and Pro-Reitoria de Pesquisa da Universidade Federal de Minas Gerais (PRPq-UFMG).

\section{REFERENCES}

1. Bhatt S, Gething PW, Brady OJ, Messina JP, Farlow AW, Moyes $\mathrm{CL}$, et al. The global distribution and burden of dengue. Nature 2013; 496:504-507. 
2. World Health Organization and the Special Programme for Research and Training in Tropical Diseases (TDR). Dengue guidelines for diagnosis, treatment, prevention and control. WHO 2013; 1-147.

3. Ministério da Saúde do Brasil (MS). Boletins Epidemiológicos da Dengue, 2013-2015; 2015. (Accessed 2015 August 06). Available at: http://portalsaude.saude.gov.br/index.php/situacao-epidemiologicadados-dengue.

4. Araújo FM, Araújo MS, Nogueira RM, Brilhante RS, Oliveira DN, Rocha MF, et al. Central nervous system involvement in dengue: a study in fatal cases from a dengue endemic area. Neurology 2012; 78:736-742.

5. Lanciotti RS, Lewis JG, Gubler DJ, Trent DW. Molecular evolution and epidemiology of dengue-3 viruses. J Gen Virol 1994; 75:65-75.

6. Figueiredo LB, Batista Cecílio A, Ferreira GP, Drumond BP, de Oliveira JG, Bonjardim CA, et al. Dengue virus 3 genotype 1 associated with dengue fever and dengue hemorrhagic fever, Brazil. Emerg Infect Dis 2008; 14:314-316.

7. Vilela AP, Figueiredo LB, dos Santos JR, Eiras AE, Bonjardim CA, Ferreira PCP, et al. Dengue virus 3 genotype I in Aedes aegypti mosquitoes and eggs, Brazil, 2005-2006. Emerg Infect Dis 2010; 16:989-992.

8. Ferreira GP, Figueiredo LB, Coelho LF, Policarpo AS, Cecilio AB, Ferreira PCP, et al. Dengue virus 3 clinical isolates show different patterns of virulence in experimental mice infection. Microbes Infect 2010; 12:546-554.
9. de Souza KP, Silva EG, de Oliveira Rocha ES, Figueiredo LB, de Almeida Leite CM, Arantes RM, et al. Nitric oxide synthase expression correlates with death in an experimental mouse model of dengue with CNS involvement. Virol J 2013; 10:267.

10. Solomon T, Dung NM, Vaughn DW, Kneen R, Thao LT, Raengsakulrach B, et al. Neurological manifestations of dengue infection. Lancet 2000; 355:1053-1059.

11. Chao DY, Davis BS, Chang GJ. Development of multiplex realtime reverse transcriptase PCR assays for detecting eight medically important flaviviruses in mosquitoes. J Clin Microbiol 2007; 45:584-589.

12. Lanciotti RS, Calisher CH, Gubler DJ, Chang GJ, Vorndam AV. Rapid detection and typing of dengue viruses from clinical samples by using reverse transcriptase-polymerase chain reaction. J Clin Microbiol 1992; 30:545-551.

13. Ju J, Ruan C, Fuller CW, Glazer AN, Mathies RA. Fluorescence energy transfer dye-labeled primers for DNA sequencing and analysis. Proc Natl Acad Sci USA 1995; 92:4347-4351.

14. Tamura K, Peterson D, Peterson N, Stecher G, Nei M, Kumar S. MEGA5: Molecular Evolutionary Genetics Analysis using Maximum Likelihood, Evolutionary Distance, and Maximum Parsimony Methods. Mol Biol Evol 2011; 28: 2731-2739.

15. Soares CN, Cabral-Castro MJ, Peralta JM, Freitas MR, PuccioniSohler M. Meningitis determined by oligosymptomatic dengue virus type 3 infection: report of a case. Int J Infect Dis 2010; 14:150-152. 